\title{
X-ray absorption near-edge structure of Ag cations in phosphate glasses for radiophotoluminescence applications
}

\author{
Hirokazu MASAI ${ }^{1 \dagger}$, Masanori KOSHIMIZU' ${ }^{2}$, Hiroki KAWAMOTO², Takahiro OHKUBO ${ }^{3}$, \\ Akitoshi KOREEDA ${ }^{4}$, Yasuhiro FUJII ${ }^{4}$, Koji OHARA ${ }^{5}$, Hironori OFUCHI ${ }^{5}$ and Hiroyuki SETOYAMA ${ }^{6}$ \\ ${ }^{1}$ Department of Materials and Chemistry, National Institute of Advanced Industrial Science and Technology, \\ 1-8-31 Midorigaoka, Ikeda, Osaka 563-8577, Japan \\ ${ }^{2}$ Department of Applied Chemistry, Tohoku University, 6-6-07 Aoba, Sendai 980-8579, Japan \\ ${ }^{3}$ Graduate School \& Faculty of Engineering, Chiba University, 1-33 Yayoi-cho, Chiba 263-8522, Japan \\ ${ }^{4}$ Department of Physical Sciences, Ritsumeikan University, Kusatsu, Shiga 525-8577, Japan \\ ${ }^{5}$ Japan Synchrotron Radiation Research Institute (JASRI/SPring-8), Kouto, Sayo-cho, Hyogo 679-5198, Japan \\ ${ }^{6}$ Kyushu Synchrotron Light Research Center (SAGA Light Source), 8-7 Yayoigaoka, Tosu, Saga 841-0005, Japan
}

X-ray absorption near-edge structure (XANES) spectroscopy is one of the most effective techniques for determining the valence states of cations. Since $K$ - and L-edge transition processes are different, the validity of $X$-ray irradiation to evaluate these excitation processes must be determined. In this study, we focus on the valence states of silver cations in aluminophosphate glasses, whose compositions have been used as radiophotoluminescence (RPL) glass detectors for personal monitoring. Slight difference was observed between the Ag K-edge XANES spectra of reference materials and those of experimental samples. It is also challenging to detect spectral changes due to the coloration of Ag-doped glass. However, absorption edge shifts depending on the valence state were observed in $\mathbf{A g ~} \mathrm{L}_{3}$-edge XANES spectra. We found that additional absorption bands, whose peak intensities increase with increasing irradiation doses, were generated at lower absorption energies during the measurement. The degrees of change in absorption intensities depend on the chemical composition of the sample. Considering the nature of the RPL glass detector, we assumed that the species generated are related to the $\mathrm{Ag}^{2+}$ species, which is an activator after irradiation.

(C2019 The Ceramic Society of Japan. All rights reserved.

Key-words : Glass, Silver, XAFS, Radiophotoluminescence, NMR, Raman, XRD

[Received September 6, 2019; Accepted October 8, 2019]

\section{Introduction}

Phosphors are equivalent to "solid luminescent materials". ${ }^{1)}$ Their lightwave conversion ability depends on the local coordination state of emission centers in the host materials. $^{2), 3)}$ For activator-doped materials, control of the valence state of activators is necessary to tailor their luminescence properties. If the host material is a crystal with an ordered structure, the local coordination state can be identified using X-ray and neutron diffraction analyses. It is much more challenging to assign the local coordination state of activators in amorphous materials because there is little structural order to fix the coordination state of the cations. For these cases, X-ray absorption fine structure (XAFS) spectroscopy is used to determine the local coordination. ${ }^{4)-6)}$ Since XAFS measurements are generally non-destructive, this technique is a powerful method for determining the valence state and the local symmetry of

\footnotetext{
Corresponding author: H. Masai; E-mail: hirokazu.masai@ aist.go.jp
}

cations, which cannot be done using other measurement techniques. Conventional XAFS measurements are performed using K-edge absorption because the extended XAFS (EXAFS) region ( $k$ range) of the K-edge is broader than that of the L-edge, in which the EXAFS region is restricted by each L-edge. By using K-edge XAFS spectroscopy, however, the observed change in the absorption edge energy, $E_{0}$, of the K-edge may be too small for the resolution of the instrument to detect, especially for heavier cations in amorphous materials. ${ }^{7)-9)}$ This is due to the heavy-atom effect and the ambiguity of the $s-p$ transition in the K-edge absorption. Recently, we demonstrated that L-edge XAFS spectroscopy can be used to more accurately determine the valence state of tin compared with $\mathrm{K}$ edge XAFS spectroscopy. ${ }^{10)}$ This result suggests that both measurements are necessary for the examination of heavy metal cations.

In the present study, we focus on silver species in phosphate glass exhibiting radiophotoluminescence (RPL). ${ }^{11-13)}$ RPL is luminescence owing to electronic transitions within luminescent centers which are generated 
in phosphors by ionizing radiation. ${ }^{14), 15)}$ Since phosphate glasses containing silver species possess good RPL, i.e., excellent response to a wide range of irradiation doses, they have been used in glass badges for personal monitoring. In the RPL mechanism reported in the literature for silver-doped phosphate glasses, $\mathrm{Ag}^{+}$cations in the glass are changed into $\mathrm{Ag}^{0}$ and $\mathrm{Ag}^{2+}$ by radiation. Since the concentrations of photoactive silver species are proportional to the irradiation dose, the Ag-doped glasses have been used as glass RPL detectors. ${ }^{16), 17)}$ Although the RPL mechanisms are proposed based on luminescence and absorption properties, there is no evidence for such valence changes in silver-doped phosphate glasses. If a disproportionation of $\mathrm{Ag}^{+}$to $\mathrm{Ag}^{0}$ and $\mathrm{Ag}^{2+}$ occurs, the valence change should be detected by the Ag XAFS measurement and is what we are attempting to confirm in this study.

In this study, we measured the valence states of $\mathrm{Ag}$ in phosphate glasses using both $\mathrm{K}$ - and L-edge XAFS analyses. Our objective was to collect direct evidence for RPL-active Ag species during the measurement. Since target materials can be used in personal monitoring equipment to detect radiation, the time-dependent change in the spectrum will be discussed by comparison with the conventional mechanism for RPL.

\section{Experimental}

\subsection{Sample preparation}

The Ag-doped aluminophosphate glasses were prepared using a conventional melt-quenching method in a platinum crucible. ${ }^{18)}$ Ammonium hydrogen phosphate $\left(\mathrm{NH}_{4}\right)_{2} \mathrm{HPO}_{4}$ and alkali carbonates were used to synthesize $\mathrm{P}_{2} \mathrm{O}_{5}$ and alkali oxides, respectively. The mixture of starting chemicals was calcined at $800^{\circ} \mathrm{C}$ for $3 \mathrm{~h}$ in a Pt crucible under an ambient atmosphere. After heat treatment, the calcined matrix was mixed with $\mathrm{Ag}_{2} \mathrm{O}$ and melted in an electric furnace at $1150^{\circ} \mathrm{C}$ for $30 \mathrm{~min}$ under an ambient atmosphere. The glass melt was quenched on a stainless-steel plate at $200^{\circ} \mathrm{C}$ and then annealed at the glass transition temperature, $T_{\mathrm{g}}$, which was measured using differential thermal analysis (DTA) for $1 \mathrm{~h}$. Commercial FD-7 glass (Chiyoda Technol Corporation) was measured as a control sample.

\subsection{Characterization}

The $T_{\mathrm{g}}$ was determined using a DTA system operated at a heating rate of $10^{\circ} \mathrm{C} \cdot \mathrm{min}^{-1}$, using a TG8120 (Rigaku, Japan). The optical absorption spectra were measured using a UH4150 spectrometer (Hitachi High-Tech. Japan). The refractive index of the glass was measured using a prism coupler (Metericon, N.J. the U.S.A.). ${ }^{31} \mathrm{P}$ and ${ }^{27} \mathrm{Al}$ magic angle spinning nuclear magnetic resonance (MAS NMR) spectra were measured to examine the local coordination state of each metal oxide. ${ }^{31} \mathrm{P}$ and ${ }^{27} \mathrm{Al}$ MAS NMR spectra of the glasses were acquired on a JEOL DELTA 600 spectrometer using a 4-mm double-resonance MAS NMR probe with a $\mathrm{ZrO}_{2}$ rotor operated at 242.9 and 156.3 $\mathrm{MHz}$. For each sample, ${ }^{27} \mathrm{Al}$ MAS NMR spectra consisting of 256 acquisitions were recorded with a pulse delay of $3 \mathrm{~s}$ and a pulse width of $0.3 \mu \mathrm{s}$ with a tip angle of $15^{\circ} .{ }^{31} \mathrm{P}$ MAS spectra were corrected with a pulse delay of $20 \mathrm{~s}$ and a 4.5- $\mu$ s $90^{\circ}$ pulse referenced against an $\mathrm{H}_{3} \mathrm{PO}_{4}$ aqueous solution at $0 \mathrm{ppm}$. The longitudinal sound velocity, $V_{\mathrm{L}}$, was calculated from $V_{\mathrm{L}}=v_{\mathrm{B}} \lambda / 2 n_{532}$, where $v_{\mathrm{B}}, \lambda, n_{532}$, are the Brillouin shift, the wavelength of incident light $(532 \mathrm{~nm})$, and the refractive index at $532 \mathrm{~nm}$, respectively. The $n_{532}$ values were calculated from the Cauchy relationship between the refractive index at different wavelengths. The Brillouin shifts, $v_{\mathrm{B}}$, of the different types of glass were measured using a high-resolution modified Sandercock Fabry-Perot system. Details of the setup are reported in a previous paper. ${ }^{19)}$ The Raman scattering analysis was also performed using a 532-nm laser, where the spectrum was recorded to a few $\mathrm{cm}^{-1}$ by employing holographic notch filters. $^{20)}$

A high-energy X-ray diffraction experiment was performed on the BL04B2 beamline at the SPring- 8 synchrotron radiation facility, using a two-axis diffractometer dedicated to the study of disordered materials. ${ }^{21)}$ The energy of the incident X-rays was $61.4 \mathrm{keV}$. The raw data were corrected for polarization, absorption, and the background, and the contribution of Compton scattering was subtracted using standard data analysis software. ${ }^{21)}$ The Ag K-edge $(25.5 \mathrm{keV})$ in the XAFS spectra were measured on the BL14B2 beamline at SPring-8 (Hyogo, Japan). The storage ring energy was operated at $8 \mathrm{GeV}$, with a current of $100 \mathrm{~mA}$. The Ag K-edge XAFS measurements were carried out using a Si (311) double-crystal monochromator in the transmission mode (Quick Scan method). Ag foil and $\mathrm{Ag}_{2} \mathrm{O}$ were measured as references. Conversely, the $\mathrm{Ag}$ $\mathrm{L}_{\mathrm{III}}$-edge $(3.35 \mathrm{keV})$ XAFS measurements were carried out on the BL11 beamline at SAGA Light Source (SAGA-LS, Saga, Japan). The spectra of the samples were measured in the fluorescence mode using 1-SSD at room temperature using a Si (111) double-crystal monochromator. The XAFS data for Ag-foil (0.001 mm), $\mathrm{AgO}$, and $\mathrm{Ag}_{2} \mathrm{O}$ were also collected in the transmission mode. According to the basic idea of X-ray absorption spectroscopy, the transmittance method is better than the fluorescence method for quantification of the valence states. However, the measurement modes of XAFS were depended on the Ag concentration. Although glass samples for Ag K-edge with the appropriate $\Delta \mu$ t value were preparable, the Ag concentration was too low to be measured by the transmission

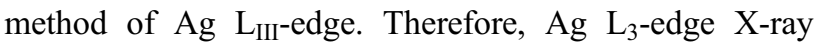
absorption near-edge structure (XANES) spectra were measured by the fluorescence method.

\section{Results and discussion}

First, we tried to quantify the valence state of $\mathrm{Ag}$ by using the Ag K-edge XANES analysis. As mentioned above, we prepared thicker samples to obtain a longer path for transmission. Using previous results, ${ }^{12), 13)}$ we calculated the chemical composition for each fraction, as shown in Table 1. The $T_{\mathrm{g}}$ for each of these glasses is also shown in Table 1. Before the measurement of Ag K-edge XAFS, 
we confirmed whether the prepared glass exhibited structure and physical properties similar to the commercial sample using different measurement techniques.

To confirm the similarity between the commercial and prepared glasses, we measured (1) optical absorption spectra, (2) refractive index, (3) ${ }^{31} \mathrm{P}$ MAS NMR, (4) ${ }^{27} \mathrm{Al}$ MAS NMR, and (5) high energy X-ray diffraction (XRD) spectra. Figure 1(a) shows the optical absorption spectra of commercial and prepared FD-7 glasses. Although small differences in the tail region of the absorption band are observed, the optical absorption edge is almost the same. Figure 1(b) shows the refractive index of commercial and prepared FD-7 glasses as a function of wavelength. The differences between the two are less than $0.2 \%$, which confirms their similarity. Figures $1(\mathrm{c})$ and 1(d) show the ${ }^{31} \mathrm{P}$ MAS (c) and ${ }^{27} \mathrm{Al}$ MAS (d) NMR spectra for these two glasses. In the ${ }^{31} \mathrm{P}$ MAS NMR spectra, the peak of the prepared FD-7 glass shifts to a higher magnetic field at 3 ppm. Considering the chemical shift of the peak position, we see that phosphorus takes the $\mathrm{Q}^{2}$ unit with a chain struc-

Table 1. Chemical compositions and glass transition temperatures $\left(T_{\mathrm{g}} \mathrm{s}\right)$ of $\mathrm{Ag}$-doped glasses. The chemical compositions were calculated using methods in a previous paper (Ref. 13)

\begin{tabular}{cccccccc}
\hline \multirow{2}{*}{ ID } & \multicolumn{7}{c}{ Chemical composition (mol \%) } \\
\cline { 2 - 6 } & $\mathrm{Ag}_{2} \mathrm{O}$ & $\mathrm{Al}_{2} \mathrm{O}_{3}$ & $\mathrm{Li}_{2} \mathrm{O}$ & $\mathrm{P}_{2} \mathrm{O}_{5}$ & $\mathrm{MgO}$ & $\mathrm{Na}_{2} \mathrm{O}$ & \\
\hline FD-3 & 1.64 & 10.24 & 27.87 & 60.25 & 0 & 0 & 426 \\
FD-5 & 0.28 & 13.35 & 0 & 63.36 & 0 & 23.01 & 480 \\
FD-6 & 0.75 & 11.73 & 0 & 61.72 & 9.25 & 16.55 & 491 \\
FD-7 & 0.09 & 13.14 & 0 & 59.04 & 0 & 27.73 & 458 \\
\hline
\end{tabular}

ture. The result of the $Q^{\mathrm{n}}$ species is reasonably assigned to the chemical composition in which the $\mathrm{P}_{2} \mathrm{O}_{5}$ fraction is over $50 \mathrm{~mol} \%$. Although the origin of the chemical shifts is unclear, we assume that a dopant that is not listed in the official composition of the FD-7 glass might affect the local coordination of the $\mathrm{P}$ units. On the other hand, there are clearly three peaks in the ${ }^{27} \mathrm{Al}$ MAS NMR spectrum. These peaks are assigned to $\mathrm{AlO}_{6 / 2}(-30--5 \mathrm{ppm}), \mathrm{AlO}_{5 / 2}$ $(-5-20 \mathrm{ppm})$, and $\mathrm{AlO}_{4 / 2}(30-50 \mathrm{ppm}){ }^{22)}$ Since the intensity normalization was performed using the $\mathrm{AlO}_{6 / 2}$ peak, a small disturbance is observed at $\mathrm{AlO}_{5 / 2}$ and $\mathrm{AlO}_{4 / 2}$. By peak deconvolution, peak areas for $\mathrm{AlO}_{6 / 2}: \mathrm{AlO}_{5 / 2}: \mathrm{AlO}_{4 / 2}$ in the commercial and prepared FD-7 glasses are $83: 13: 4$, and 74:20:6, respectively. Some structural differences are observed in the ${ }^{27} \mathrm{Al}$ MAS NMR spectrum. We prepared a high-energy XRD spectrum to check the differences. Figure 1(e) shows the structural factors, $S(Q)$, for the two glasses. Figure 1(f) shows the total correlation function, $T(r)$, for the two glasses that were obtained from the Fourier transform of the $S(Q)$. Peaks at $1.54,1.89$, and $2.50 \AA(1 \AA=0.1 \mathrm{~nm})$ are assigned to the $\mathrm{P}-\mathrm{O}, \mathrm{Al}-\mathrm{O}$, and $\mathrm{Na}-\mathrm{O}$ bonds, respectively. ${ }^{23), 24)}$ Although a small peak is observed around $2 \AA$ (close to the Al-O bond) in the commercial FD-7 glass sample, the differences cannot be explained by the types of $\mathrm{AlO}_{x}$ groups (terminating or bridging unit). ${ }^{25)}$ These results, therefore, indicate that a small amount of a dopant exists in the glass. The addition of tiny amounts of dopants in the commercial FD-7 glass sample is also suggested by differences in the Raman spectra. Figures 2(a) and 2(b) are Raman spectra of commercial and prepared FD-7 glasses with HH and HV polarizations, (a)

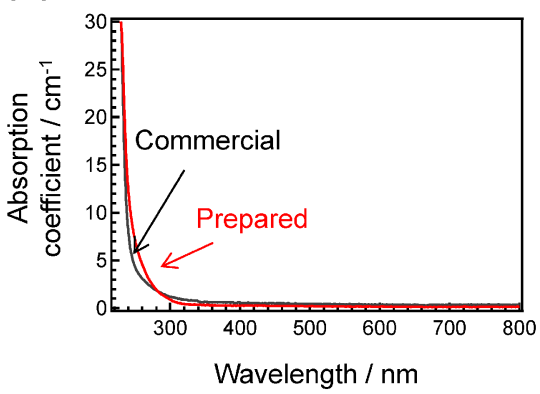

(d)

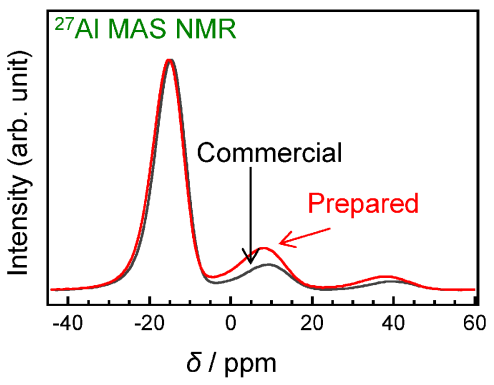

(b)

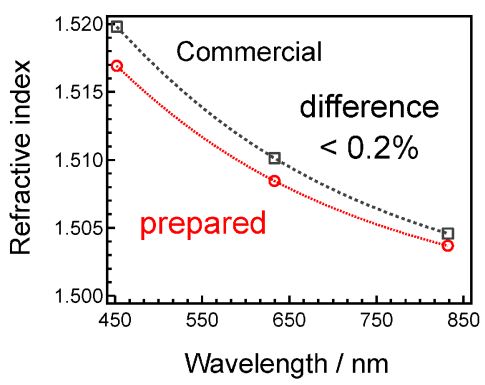

(e)

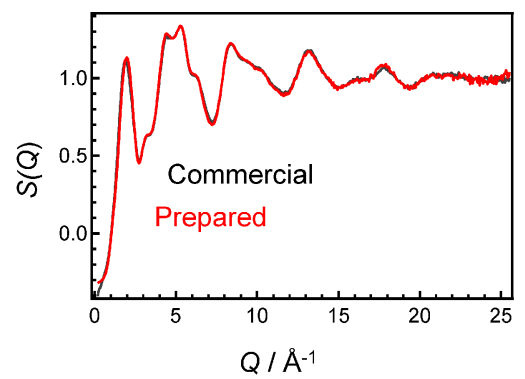

(c)

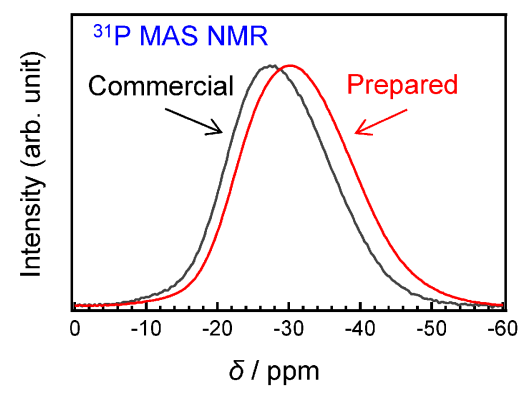

(f)

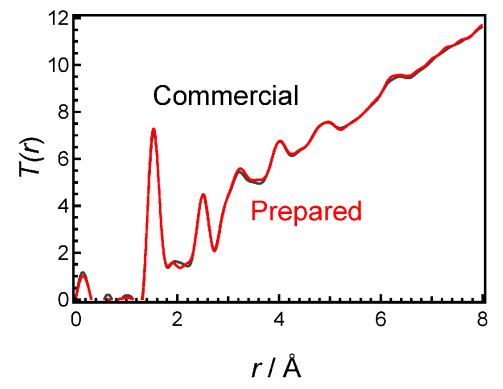

Fig. 1. Reproductivity of commercial and prepared FD-7 glasses. (a) Optical absorption spectra, (b) refractive index, (c) ${ }^{27}$ Al MAS NMR spectra, (d) ${ }^{31}$ P MAS NMR spectra, (e) $S(Q)$, and (f) $T(r)$ of commercial and prepared FD-7 glasses. 
(a)

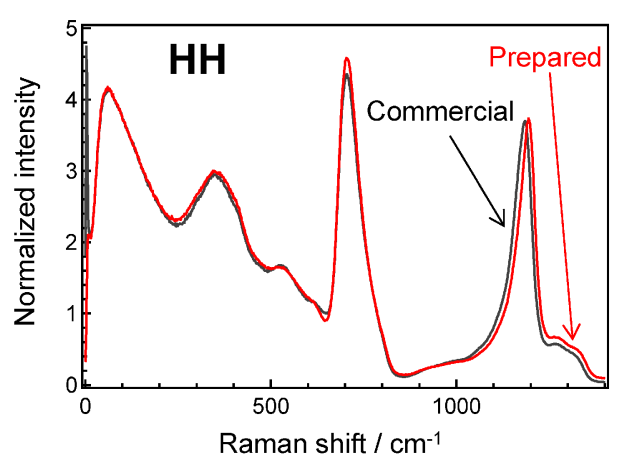

(b)

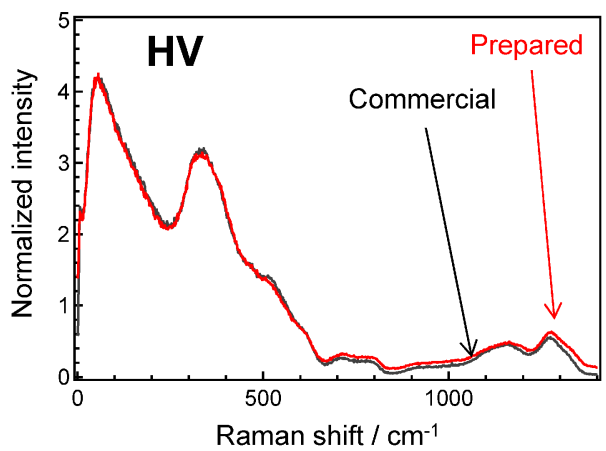

Fig. 2. Comparison of Raman spectra between commercial and prepared FD-7 glasses. (a) HH and (b) HV polarizations.

(a)

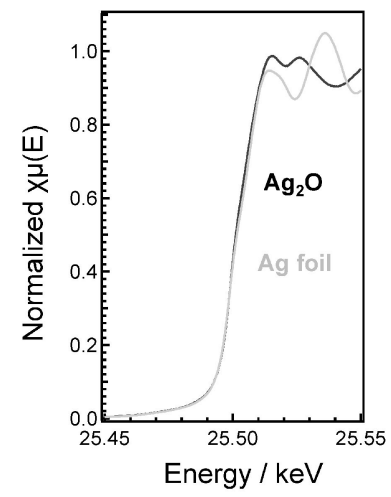

(b)

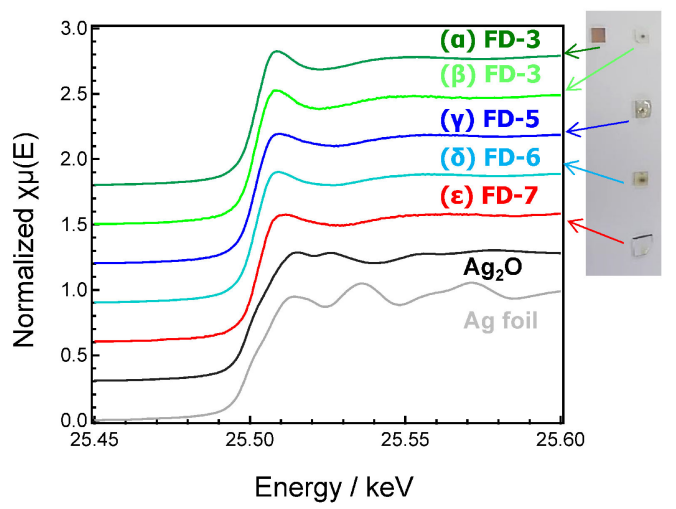

Fig. 3. Valence state of silver in phosphate glasses. (a) $\mathrm{Ag} \mathrm{K}$-edge XANES spectra of $\mathrm{Ag}$ foil and $\operatorname{Ag}_{2} \mathrm{O}$ and (b) Ag K-edge XANES spectra of several Ag-doped phosphate glasses along with those of Ag foil and $\mathrm{Ag}_{2} \mathrm{O}$.

respectively. In the lower frequency region, a boson peak ${ }^{26}$ is observed in both glasses. Notably, the boson peaks are almost identical, suggesting that boson-peak-related structures in both glasses are the same. On the contrary, there are clear differences observed in the higher frequency region. Although the spectral shapes are similar, the frequency of $\mathrm{P}-\mathrm{O}-\mathrm{P}$ stretching ${ }^{27)}$ in the prepared FD-7 glass seen at 700 $\mathrm{cm}^{-1}$ is higher than that in the commercial sample, indicating that the oscillation strength of the prepared sample is stronger than that of the commercial sample. Also, a small frequency shift is observed in the vibration mode at 1200 $\mathrm{cm}^{-1}$, due to $\mathrm{PO}_{2}$ stretching. Although slight differences between the commercial and prepared FD-7 glasses are found in the $T_{\mathrm{g}}$, refractive index, and longitudinal elastic modulus (see Table 2), we assume that this series of results is enough to conclude that the prepared FD-7 glass behaves the same as the commercial glass. Therefore, we prepared several Ag-doped phosphate glasses, shown in Table 1, for the measurement of Ag K-edge XAFS spectra.

Figure 3(a) shows the Ag K-edge XANES spectra of $\mathrm{Ag}$ foil and $\mathrm{Ag}_{2} \mathrm{O}$. Since the measurement resolution is $\Delta \mathrm{E} / \mathrm{E}=\sim 6 \times 10^{-5}$, we conclude that these two chemicals possess very similar absorption edges, and that quantitative analysis of the Ag species from the absorption edge energy of the K-edge XANES spectra is challenging to
Table 2. Comparison of physical properties of prepared FD-7 glass with that of commercial glass. ( $T_{\mathrm{g}}$ is the glass transition temperature.)

\begin{tabular}{cccc}
\hline & $T_{\mathrm{g}} /{ }^{\circ} \mathrm{C}$ & Density $/ \mathrm{g} \cdot \mathrm{cm}^{-3}$ & Elastic modulus $c_{11} / \mathrm{GPa}$ \\
\hline Commercial & 450 & 2.60 & 79.2 \\
Prepared & 458 & 2.59 & 77.9 \\
\hline
\end{tabular}

calculate. This difficulty also exists for the study of Agdoped phosphate glasses. Figure 3(b) shows Ag K-edge XANES spectra of several Ag-doped phosphate glasses, whose chemical compositions are listed in Table 1, along with those for $\mathrm{Ag}$ foil and $\mathrm{Ag}_{2} \mathrm{O}$. A photograph of these glasses is shown on the right side. The color centers at the irradiation spot are visible in all glasses. Surprisingly, these spectra exhibit almost the same spectral shape, although the chemical composition of each host glass is different. When we compare the spectra of colored FD3 $(\alpha)$ with non-colored FD3 $(\beta)$, we find that there is little difference between the two. Although coloration originates from the precipitation of Ag nanoparticles ${ }^{28)}$ no absorption band exists for their formation.

On the other hand, we also measured Ag K-edge XANES spectra for longer times. Spectra changes were also undetectable despite the apparent color change at the 
(a)

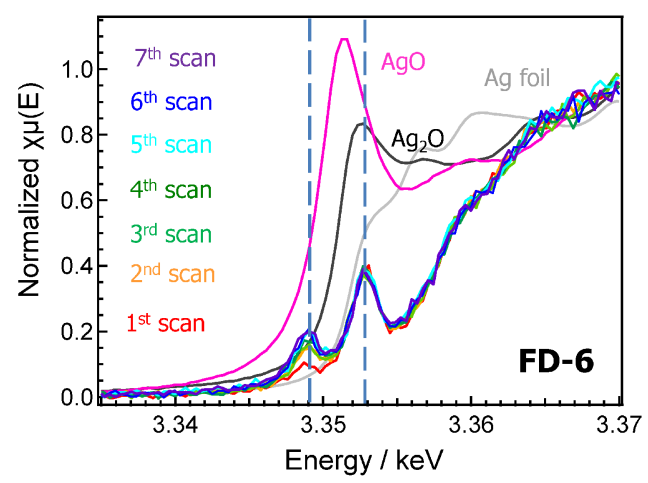

(b)

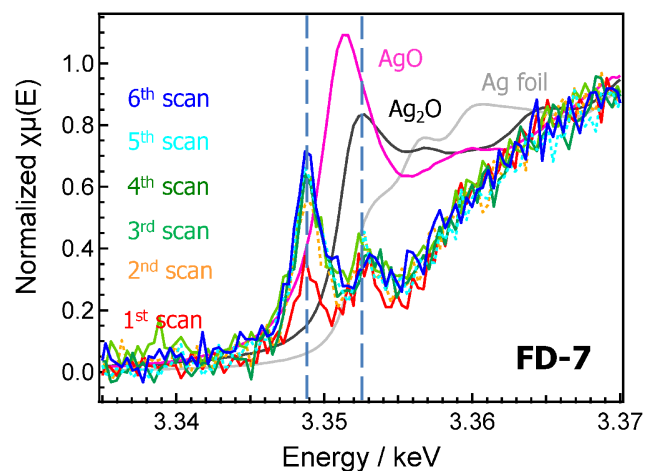

Fig. 4. Ag $\mathrm{L}_{\mathrm{III}}$-edge XANES spectra. (a) Prepared FD-6 and (b) FD-7 glasses depending on the number of scans.

(a)

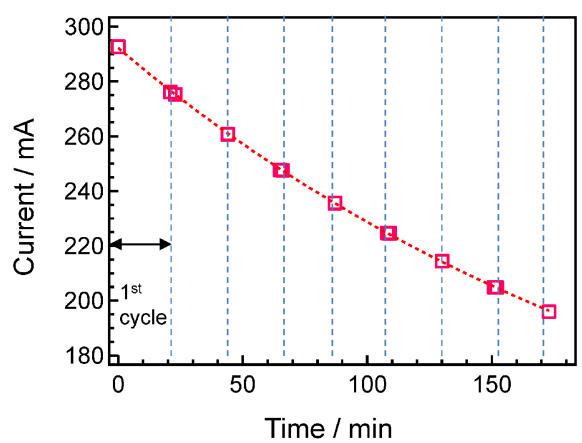

(b)

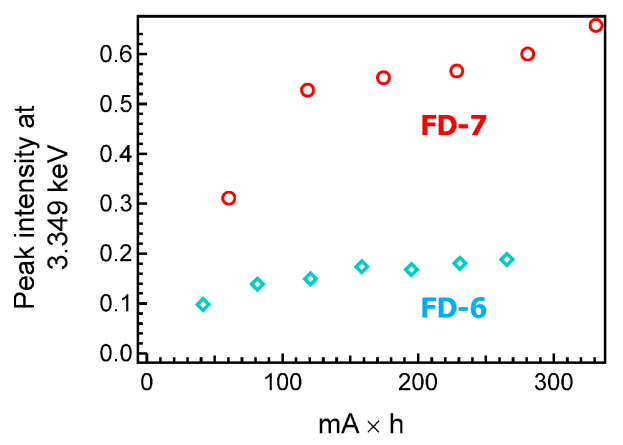

Fig. 5. (a) Decay of electric current during the irradiation of one day, (b) generated absorption peak intensity at $3.349 \mathrm{keV}$ of prepared FD-6 and FD-7 glasses as a function of electrical current multiplied by time.

irradiation spot of the sample. Based on these data, we conclude that it is difficult to determine the valence state of doped silver using K-edge XAFS analysis.

Since spectral changes should occur due to the formation of RPL $\left(\mathrm{Ag}^{2+}\right.$ and $\left.\mathrm{Ag}^{0}\right)$ centers, $\mathrm{Ag} \mathrm{L}$-edge XANES spectra were generated with repeat scans to check their time-dependent change. Figures 4(a) and 4(b) show the Ag $\mathrm{L}_{3}$ edge XANES spectra of prepared FD-6 and FD-7 glasses after different irradiation doses. Three references are shown for comparison ( $\mathrm{Ag}$ foil, $\mathrm{Ag}_{2} \mathrm{O}$, and $\mathrm{AgO}$ ). In contrast to the Ag K-edge XANES spectra, whose s-p transition path becomes obscured with an increase in the atomic number, visible differences due to the valence state of Ag species are observed between them. Since spectral signal-to-noise ratios reflect the concentration of $\mathrm{Ag}$, smooth spectra are observed for prepared FD-6 glasses. The energy of the white lines of the FD- 6 and FD-7 glasses with small irradiation doses are located at the $\mathrm{Ag}_{2} \mathrm{O}$ peak, indicating that the $\mathrm{Ag}$ species before irradiation is monovalent. Notably, the absorption band at $3.349 \mathrm{keV}$ was generated in these glasses by increasing irradiation doses. Although the peak intensity at $3.349 \mathrm{keV}$ increases as the number of scans increases, no energy shift of the peak is seen. Also, it is notable that the only generated absorption band is changed despite a similar shape of the remaining part. Moreover, the energy of the absorption band is almost the same independent of the chemical composition of the host glass. The reason will be discussed later in this paper.

It is also notable that the absorption edge shifts to lower energy as the valence state of silver increases. This is an unusual trend for common cations, which is also observed in a previous paper. ${ }^{29)}$ Also, others show that the spectral shape of silver can be drastically changed depending on the covalency and symmetry of the silver cation. ${ }^{30)}$ Therefore, we conclude that the $\mathrm{L}_{3}$-edge XANES spectra are suitable for the evaluation of the local coordination state and valence state of doped silver in amorphous glasses.

We should also note that the normalization of spectra is necessary because storage ring current of SAGA-LS decreased over time, as shown in Fig. 5(a). Hence, in this study, the electric current multiplied by time is used for our calculations. Figure 5(b) shows the absorption peak intensity at $3.349 \mathrm{keV}$ for the prepared FD-6 and FD-7 glasses as a function of electric current multiplied by time. These data are plotted without baseline correction. It was reported that valence changes of cations in glass by a synchrotron X-ray beam is seen better at lower dopant concentrations than that at higher dopant concentration. ${ }^{31)} \mathrm{We}$ also note that the concentration of generated species in both glasses is similar because the level of silver in the FD-6 
glass is higher than that in the FD-7 glass. Although the $T_{\mathrm{g}}$ might affect the radiation-induced color center formation, there is no direct correlation between the local coordinations of silver cations and the macroscopic physical properties. This point-of-view will be clarified in a separate paper.

Here, we discuss the origin of peaks by comparison with the conventionally proposed mechanism. Based on our results, the spectral changes caused by irradiation suggest that new Ag species are generated by X-ray irradiation. Since there is no significant difference in absorption energy between the two glasses, we expect that changes occur in the vicinity of large mole fractions of phosphate units. If a change from $\mathrm{Ag}^{+}$to $\mathrm{Ag}^{2+}$ and $\mathrm{Ag}^{0}$ occurs according to the conventional mechanism, ${ }^{16), 17)}$ the change of the peak around XANES should be visible. However, differential spectra after subtraction of the original spectrum show that changes cannot be confirmed anywhere along the entire energy range. The results clearly indicate that not all $\mathrm{Ag}^{+}$species change by X-ray irradiation. Recently, McKeever et al. proposed the presence of Ag cluster in the RPL glass and it is the main origin of RPL instead of divalent state of $\mathrm{Ag}$, based on electron spin resonance studies. ${ }^{32)}$ However, as mentioned above, remarkable spectra change by irradiation is only observed at $3.349 \mathrm{keV}$. From the present XANES study, we cannot confirm the existence of Ag clusters.

One thing we have to emphasize is that the energy of the generated absorption band is located at lower energy than that of $\mathrm{AgO}$. Considering the trend of absorption energy in Ag species, peaks might be assigned to Ag species higher than 2. A peak might correspond to the conventional RPL center since changes in other Ag species cannot be detected. For a better understanding, we recorded XANES spectra using different references. An approach might be comparing the valence state of silver with a result of another element belonging to the same group. We have recently reported valence states of $\mathrm{Cu}$-doped phosphate glasses, whose chemical compositions are the same as in Table $1 .{ }^{33)}$ In these glasses, almost all $\mathrm{Cu}$ cations were found to take divalent states by the $\mathrm{Cu}$ K-edge XANES analysis. ${ }^{33)}$ Therefore, we expect that stable valence states of $\mathrm{Cu}$ and $\mathrm{Ag}$ in the same glasses will be different. Asakura et al. proposed that the $\mathrm{d}^{10)}$ state will exhibit a transition from $2 p$ to $5 \mathrm{~s}$ enhanced by the $\mathrm{s}-\mathrm{d}$ hybridization in the XANES spectra. ${ }^{30)}$ Since we expect that transitions might be affected by the glass system, i.e., the local coordination state of the cation and electrons, we have to do precise experiments based on several variables. From the present results, we find the following; (1) not all Ag species show an X-ray-induced change, (2) generated Ag species take a divalent, or higher valence state, and (3) degrees of change might be correlated with the host glasses. Since it is found that Ag L-edge XANES analysis is effective to discuss the valence change of silver after X-ray irradiation, we will perform the XANES analysis using commercial RPL glasses in the near future. We emphasize that the present approach will contribute to a deeper understanding of the local coordination of useful activators in phosphor application.

\section{Conclusion}

We have examined the valence states of $\mathrm{Ag}$ cations in phosphate glasses using $\mathrm{K}$ - and $\mathrm{L}_{3}$-edge XANES measurements. Since it is hard to detect a difference between $\mathrm{Ag}$ metal and $\mathrm{Ag}^{+}$in $\mathrm{Ag} \mathrm{K}$-edge absorption spectra, we conclude that evaluation of the valence state of doped $\mathrm{Ag}$ species is difficult, even though the formation of color centers is clearly visible after X-ray irradiation. Conversely, differences due to the valence state of Ag species are found in the $\mathrm{L}_{3}$-edge XANES analysis. In contrast to conventional elements, the peak energy of the white line shifts toward lower energy as the oxidation state increases. It should be noted that an absorption peak is generated during irradiation and that the intensity increases with increasing irradiation doses. Although the origin of these peaks is not yet fully understood, we expect that the generated peak strongly correlates with the $\mathrm{Ag}^{2+}$ species, which is thought to be at the emission center of the RPL phenomena. Our findings will lead to a better understanding of the RPL behavior in glass and to the design of excellent RPL glass detectors in the future.

Acknowledgments This work was partially supported by the Japan Society for the Promotion of Science Grantin-Aid for Scientific Research (B) Number 18H01714. High energy XRD measurements were performed on the BL04B2 at SPring- 8 with the approval of the Japan Synchrotron Radiation Research Institute (JASRI) (Proposal No. 2018A1309). The Ag K-edge XAFS measurements were performed on the BL14B2 at SPring-8 with the approval of the JASRI (Proposal No. 2018A1557). The Ag L-edge XAFS measurements were performed on the BL11 at SAGA-LS with the permission of the Kyushu Synchrotron Light Research Centre (Proposal No. 1806052F and 1809068F). The author (H.M.) thanks Prof. S. Hosokawa (Kyoto University) for many fruitful discussions.

\section{References}

1) Phosphor Handbook 2nd Edition, Ed. by W. M. Yen, S. Shionoya and H. Yamamoto, CRC Press, Boca Raton, USA (2007).

2) G. Blasse, Prog. Solid State Ch., 18, 79-171 (1988).

3) C. Feldmann, T. Justel, C. R. Ronda and P. J. Schmidt, Adv. Funct. Mater., 13, 511-516 (2003).

4) F. Farges, Y. Lefrere, S. Rossano, A. Berthereau, G. Calas and G. E. Brown, J. Non-Cryst. Solids, 344, 176188 (2004).

5) I. Nakai, C. Numako, H. Hosono and K. Yamasaki, J. Am. Ceram. Soc., 82, 689-695 (1999).

6) P. M. Peters and S. N. Houde-Walter, Appl. Phys. Lett., 70, 541-543 (1997).

7) F. Fayon, C. Landron, K. Sakurai, C. Bessada and D. Massiot, J. Non-Cryst. Solids, 243, 39-44 (1999).

8) M. R. Antonio, L. Soderholem and I. Song, J. Appl. Electrochem., 27, 784-792 (1997).

9) F. J. Espinosa, J. M. de Leon, S. D. Conradson, J. L. Pena and M. Zapata-Torres, Phys. Rev. Lett., 83, 3446- 
3449 (1999).

10) H. Masai, T. Ina and K. Mibu, Sci. Rep., 8, 415 (2018).

11) R. Yokota and H. Imagawa, J. Phys. Soc. Jpn., 20, 1537-1538 (1965).

12) R. Yokota and H. Imagawa, J. Phys. Soc. Jpn., 23, 1038-1048 (1966).

13) R. Yokota, Oyo Butsuri, 40, 1292-1307 (1971) [in Japanese].

14) J. H. Schulman, R. J. Ginter and C. C. Klick, J. Appl. Phys., 22, 1479-1487 (1951).

15) J. H. Schulman and H. W. Etzel, Science, 118, 184-186 (1953).

16) Y. Miyamoto, Y. Takei, H. Nanto, T. Kurobori, A. Konnai, T. Yanagida, A. Yoshikawa, Y. Shimotsuma, M. Sakakura, K. Miura, K. Hirao, Y. Nagashima and T. Yamamoto, Radiat. Meas., 46, 1480-1483 (2011).

17) H. Kawamoto, Y. Fujimoto, M. Koshimizu, G. Okada, T. Yanagida and K. Asai, Jpn. J. Appl. Phys., 57, 062401 (2018)

18) H. Masai, T. Tanimoto, T. Fujiwara, S. Matsumoto, Y. Takahashi, Y. Tokuda and T. Yoko, J. Non-Cryst. Solids, 358, 265-269 (2012).

19) Y. Onodera, S. Kohara, H. Masai, A. Koreeda, S. Okamura and T. Ohkubo, Nat. Commun., 8, 15449 (2017).

20) Y. Fujii, D. Katayama and A. Koreeda, Jpn. J. Appl. Phys., 55, 10TC03 (2016).

21) S. Kohara, M. Itou, K. Suzuya, Y. Inamura, Y. Sakurai,
Y. Ohishi and M. Takata, J. Phys. Condens. Matter, 19, 506101 (2007).

22) S. Sen, Z. Xu and J. F. Stebbins, J. Non-Cryst. Solids, 226, 29-40 (1998).

23) K. Suzuya, D. L. Price, C.-K. Loong and S. W. Martin, J. Non-Cryst. Solids, 232-234, 650-657 (1998).

24) S. Ansell, S. Krishnan, J. K. R. Weber, J. J. Felten, P. C. Nordine, M. A. Beno, D. L. Price and M. L. Saboungi, Phys. Rev. Lett., 78, 464-466 (1997).

25) C. J. Benmore, J. K. R. Weber, S. Sampath, J. Siewenie, J. Urquidi and J. A. Tangeman, J. Phys. Condens. Matter, 15, S2413-S2424 (2003).

26) M. D. Ediger, C. A. Angell and S. R. Nagel, J. Phys. Chem., 100, 13200 (1996).

27) A. K. Yadav and P. Singh, RSC Adv., 5, 67583 (2015).

28) M. D. Malinsky, K. L. Kelly, G. C. Schatz and R. P. Van Duyne, J. Phys. Chem. B, 105, 2343-2350 (2001).

29) P. Behrensa, S. Aûmannb, U. Bilowc, C. Linkec and M. Jansenc, Z. Anorg. Allg. Chem., 625, 111-116 (1999).

30) T. Miyamoto, H. Niimi, Y. Kitajima, T. Naito and K. Asakura, J. Phys. Chem. A, 114, 4093-4098 (2010).

31) P. G. Ferreira, D. de Ligny, O. Lazzari, A. Jean, O. C. Gonzalez and D. R. Neuville, Chem. Geol., 346, 106112 (2013).

32) S. W. S. McKeever, S. Sholom and N. Shrestha, Radiat. Meas., 123, 13-20 (2019).

33) H. Masai, H. Ofuchi, G. Okada, N. Kawaguchi and T. Yanagida, Sens. Mater., 31, 1297-1303 (2019). 